\title{
REPOSITORY CORTICOTHERAPY IN OPHTHALMIC THEORY AND PRACTICE*
}

\author{
BY \\ F. C. RODGER \\ From the Princess Margaret Hospital, Swindon, Wilts.
}

IN repository corticotherapy a quantity of long-acting steroid is placed directly into the tissues; in ophthalmology, by reason of the delicacy of the tissues concerned, the steroid preparation used must not cause any damage when it is forced into the tissues, or later, and at the same time must retain its potency throughout the period of absorption. It is claimed by the manufacturers that Depo-Medrone (methylprednisolone acetate, Upjohn) fulfils these criteria. Clearly, this type of treatment will have a number of advantages in ophthalmic practice; for example, it will be preferable where locally administered steroids are impracticable, or when systemic steroids are dangerous by reason of systemic disease or emotional instability. These points will be enlarged upon later.

\section{Methods of Administration}

Depo-Medrone (or Depo-Medrol, as it is called in the U.S.A.) can be injected in a variety of ways. Some workers have placed it in the eye under the bulbar conjunctiva in the region of the lower fornix (Gebertt, 1961), others, after topical anaesthesia, into the upper temporal quadrant of the bulb 10-14 mm. from the limbus under cover of the upper lid (Coles, Krohn, Breslin, and Braunstein, 1962). Bonnet (1962) believes that it should be a deep injection under Tenon's capsule, not a simple subconjunctival injection. Klodell and Bloomberg (1964) prefer the retrobulbar route to any other, a technique used earlier by Gebertt (1961), and by subsequent workers, selectively. Klodell and Bloomberg (1964), however, claim that there is less discomfort to the patient when the retrobulbar route is used, the eye is not disfigured, and the patient accepts the thought of the injection more readily, as it appears to be a more meaningful procedure and its administration by this route is less frightening than by the subconjunctival.

All these techniques were used in the present study, including the placement of DepoMedrone directly into the subcutaneous tissues of the lids through the skin, either deep to the lower fornix, or adjacent to the lacrimal passages, as indicated; it was also placed directly into the extra-ocular muscles and inside the tear sac in certain other selected cases.

In general, I have found it better to use bulbar subconjunctival injections in the lower fornix (after topical anaesthesia) for inflammations of the anterior segment, because the surgeon is then able to observe the slow dissolution of the drug, and because it is an easy and quick technique, less troublesome to the patient than when placed under the upper lid, where

\footnotetext{
* Received for publication September 18, 1964.
} 
it is subsequently more readily felt as a foreign body; in addition, it is less painful than an injection placed under Tenon's capsule, and is just as effective. As an alternative it may be placed below the conjunctiva in the lower fornix through the skin of the lower lid.

When, on the other hand, there is inflammation of the posterior segment, or of the ciliary body, retrobulbar injections have proved to be better; these injections are given as in local anaesthesia for cataract through a long needle into the apex of the muscle cone behind the eyeball; following the injection of the anaesthetic of choice, the needle is left in situ in the orbit and Depo-Medrone then drawn into the syringe and injected down the original needle.

Direct injection into the lids or into and around the lacrimal passages has also proved highly effective, as will be shown. The technique is straightforward and needs no discussion.

\section{Preparation used and its Storage}

The preparation used in these experiments was a repository form of methylprednisolone acetate available commercially as Depo-Medrone. Its composition is as follows:

$\left.\begin{array}{lr}\text { Methylprednisolone acetate } & 40 \mathrm{mg} . \\ \text { Polyethylene glycol 4,000 } & 29 \mathrm{mg} . \\ \text { Sodium chloride } & 8 \cdot 7 \mathrm{mg} . \\ \text { Myristyl-gamma-picolinium chloride }(1: 5,000) & 0 \cdot 19 \mathrm{mg} .\end{array}\right\}$ Per ml.

As repeated cooling to refrigerator temperature and warming to room temperature will upset the physical properties of the suspension, leading to crystal formation, storage in a refrigerator is not advised and the ampoules used were kept at room temperature.

\section{Selection of Dosages}

When $0 \cdot 15 \mathrm{ml}$. of Depo-Medrone is injected under the bulbar conjunctiva, it can be seen in that position for about three weeks, while its size keeps diminishing until it is totally absorbed. From the observations made, it would appear that a dosage of $0.05 \mathrm{ml}$. will remain in situ for about seven days; thus, a retrobulbar injection of $0.50 \mathrm{ml}$. should remain active for about ten weeks. It has been our custom, nevertheless, to allow roughly half this period and twice this dosage, in the case of retrobulbar injections, before assessing the effect and repeating the treatment if thought fit; at the same time the possibility remains that more frequent injections may sometimes be indicated.

Gebertt (1961) repeated his retrobulbar injections of $0.50 \mathrm{ml}$. every three or four weeks, which roughly agrees with the observations made here. Klodell and Bloomberg (1964) administered $1.0 \mathrm{ml}$. by the retrobulbar route at various periods, sometimes as frequently as every fortnight; this frequency would seem to be rather high and not without some slight risk. Coles and others (1962) pointed out that with repeated injections at the same subconjunctival site scarring occurs, so that different quadrants of the bulb require to be used; this is perhaps less likely to happen within the muscle cone behind the eyeball, but we do not know, and as long as we do not know, it would appear unwise to administer too much too often. It is possible that quite a large quantity of the suspension might accumulate and produce pressure symptoms, if given too frequently. Klodell and Bloomberg (1964) claim that with $1.0 \mathrm{ml}$. injections, momentary diplopia and ptosis of a somewhat longer duration are sometimes witnessed, although recognizable retrobulbar haemorrhages (presumably 
large ones) did not occur, and the patients never complained. This did not occur in the present study. Our patients liked the initial injection of a local anaesthetic no better than the initial injection of Depo-Medrone without an anaesthetic, but with 1-2 per cent. Lignocaine there is no after-pain; without the local anaesthetic, occasionally there is. Thus, it is recommended that $0.50 \mathrm{ml}$. of 2 per cent. Lignocaine (with or without adrenaline) should be injected by the retrobulbar route to be followed straight away by $1.00 \mathrm{ml}$. of Depo-Medrone, and that this treatment should not be repeated until a month has elapsed unless there is a very strong indication for it. For all other types of repository corticotherapy, the dosage used in the bulbar subconjunctival route, namely $0.15 \mathrm{ml}$., is recommended. As long as the drug can be seen with the naked eye, of course, there is no need to repeat the therapy; when it cannot be seen (as when under the skin) then an injection of $0.15 \mathrm{ml}$. should be repeated every three weeks until the condition settles.

\section{Analysis of Depo-Medrone Deposit after 21 Days in a Human Eye}

A $302 \mu \mathrm{g}$. chalky deposit was recovered for analysis from under the conjunctiva of the eye of a volunteer who had received a $0.25 \mathrm{ml}$. subconjunctival injection of DepoMedrone 21 days previously.

At least $125 \mu \mathrm{g}$. (41 per cent.) of this material was unequivocally identified as unchanged methylprednisolone acetate by thin-layer chromatography, and by infrared and ultra-violet spectroscopy; $98 \mu \mathrm{g}$. (33 per cent.) of the material was ocular connective tissue. Part of the remaining $79 \mu \mathrm{g}$. ( 26 per cent.) could be attributed to non-quantitation of methylprednisolone acetate, although at least one other organic component was present whose amount was insufficient for identification. There was no detectable methylprednisolone (free alcohol).

Thus there is no doubt that the drug remains active as long as it is present, and does not become changed or inert after deposition.

\section{Effect of Repository Corticotherapy on the Surrounding Tissues}

\section{Tenon's Capsule}

A fragment of Tenon's capsule removed fourteen days after the original injection and in which a few Depo-Medrone particles were embedded was processed between two pieces of liver tissue as it was so minute. Sections were stained with Lillie's haematoxylin and eosin and Gridley's modification of the quadruple stain. The tissue showed no cellular or vascular evidence of inflammation. In all respects the portion of Tenon's capsule was normal.

\section{Extrinsic Ocular Muscle}

Depo-Medrone was injected through the conjunctiva into the belly of a rectus muscle during a bilateral resection; one muscle only was resected. Fourteen days later the other rectus into which Depo-Medrone had been injected was also resected and the piece of muscle, approximately 5-6 $\mathrm{mm}$. in length, 3-4 mm. wide, and 1-2 $\mathrm{mm}$. thick was submitted for histological examination. Lillie's haematoxylin and eosin staining was carried out. Microscopic examination revealed a non-stainable spicule (nature unknown) embedded in the muscle fragment; it was approximately 
$10 \mu$ wide and $100 \mu$ long. A mild infiltration of inflammatory cells was present in the immediate area. The reaction was not typically foreign body in type. Elsewhere the muscle appeared normal except for some perivascular haemorrhages which were regarded as surgically induced. Thus it is unlikely that the presence of DepoMedrone produces much reaction in muscle; in fact, it is likely to be no more than that caused by the hypodermic needle. Despite this, the view that retrobulbar injections of the drug should not be used too frequently in too large doses gains some support from these findings, especially as there is the added risk of haemorrhage.

Recently, Flandre, Damon, Weisbuch, Peillex, François, and Ferran (1963) have carried out somewhat similar experimental studies on rat muscle. Fifteen days after the subcutaneous implantation of a foreign body and an intramuscular injection of $4 \mathrm{mg}$. of methylprednisolone acetate, a portion of the underlying muscle was excised, demonstrating the complete inhibition of the usual foreign-body reaction.

\section{Advantages of Repository Corticotherapy}

The most obvious of the practical advantages have been mentioned in the introduction, especially those where systemic steroids are dangerous: there are others. In agricultural areas, where frequent attendances are not always convenient because of infrequent transport services, this form of therapy can be a boon; it is invaluable in the case of the elderly or incapacitated patient who relies on a neighbour to insert drops, and who is unable to insert them himself in the absence of such a helper. Many old people insert eye drops indifferently, no matter whether they apply them to their own eyes or to their spouse's; this is the real reason why the condition does not appear to respond to topical steroid therapy and is an indication for the repository technique. Unco-operative patients of all ages who cannot remember, or cannot be bothered, to insert drops, will also benefit.

Apart from these obvious practical considerations, there are more important aspects to repository therapy, as it is claimed to be more effective than topical application and safer than oral administration. The former claim is difficult to prove, although one may suspect it when the patient remarks on the speed of recovery when the technique has been used to treat a relapse. There is little doubt that the second claim is a fair one, namely, that it is a safer form of therapy than systemic administration (Coles and others, 1962). The long-term use of systemic steroids not infrequently results in undesirable side-effects such as moon-face, obesity, hirsutism, decalcification, and mental depression; more recently we have heard it reported from several sources that cataract may also be listed as a complication (Crews, 1963). Diabetes, peptic ulcer, hypertension, and emotional instability are, without doubt contra-indications to systemic therapy, and yet repository therapy in such instances is quite safe.

From all the information we possess, the advantages of injecting a long-acting steroid locally may be summed up in the following way:

(1) It is more effective than topical therapy, as the drug's suppressive action is constant during the day and night; in this respect it is to be preferred as an alternative.

(2) It is possible to inject the steroid preparation into locations inaccessible to topical application, thus avoiding the expense and side-effects of systemic administration. 
(3) Because it inhibits hypersensitivity reactions to antigen-antibody complexes, provided that it is not used in large doses and provided (as will be shown) that it is not placed directly on or in the affected focus (thereby giving a high local titre) but "off target"-which is easily done with repository therapy-it may be used successfully against certain diseases where it is generally believed to be contra-indicated.

\section{Results}

\section{As a Better Alternative to Topical Therapy}

The treatment has been found effective against both granulomatous and nongranulomatous anterior uveitis, perhaps more so against the latter, or when mixed uveitis occurs. It has now become my practice to give a subconjunctival injection of Mydricaine (No. 2) and Depo-Medrone directly into the lower fornix as soon as a diagnosis of anterior uveitis has been made, following this with atropine drops only. As a rule the inflammatory lesion disappears with one injection, although a subsequent follow-through with topical steroids or a second injection may occasionally be needed; thus it is not an expensive form of therapy. There are so many causes of anterior uveitis that one should expect occasional disappointments with corticotherapy, although this was seldom experienced. When it is unsuccessful one should look for some cause other than the apparent; for example, a case of mixed anterior uveitis associated with, and believed to be caused by, ankylosing spondylitis, which did not yield either to subconjunctival or retrobulbar injections of Depo-Medrone, subsequently proved to be a case of Reiter's disease. In this way, therapeutic trials involving this technique can help ophthalmic theory as well as practice.

Anterior uveitis is the principal lesion of the anterior segment where this type of treatment is indicated, but it has also been used with effect against rosacea keratitis and in lime burns. A single case of sympathetic ophthalmia dragged on for weeks with topical therapy and disappeared with two Depo-Medrone subconjunctival injections separated by a three-week interval. It has been found to have no effect against ocular pemphigus; on the other hand, it quickly controlled a membranous conjunctivitis in an early case of Stevens-Johnson disease.

Coles and his colleagues (1962) have recommended the use of repository corticotherapy as a routine during glaucoma surgery, not just to reduce post-operative inflammation but to prevent closure of the fistulizing tract by inhibiting fibrosis. The results of anterior flap sclerotomy with basal iridencleisis, however, seem to be so good with topical therapy that subconjunctival repository therapy appears to be an unnecessary luxury. In 32 patients in 1963 I had only one case where excessive healing closed the anterior flap; this happened twice in the same eye and the second time was not prevented by subconjunctival administration of Depo-Medrone. Success was achieved at a third operation by a stricter control of bleeding points and by cauterizing freely the adjacent blood vessels, achieving a higher degree of avascularity before replacing the conjunctival flap.

In cataract surgery, repository corticotherapy is contra-indicated because it impedes fibroblast formation. It has been found useful, on the other hand, in certain complications; after using alpha-chymotrypsin, if the lens has to be tumbled to be removed, it is known that a striate keratitis will all too frequently result (Boyd, 
1960). Depo-Medrone, therefore, was deliberately administered under the bulbar conjunctiva after tumbling had been performed in two such instances; in both there was a slight keratitis but it was extremely mild and lasted only a couple of days. The other complication of cataract surgery, in which it is believed that this kind of therapy is helpful, is when a hypermature cataract unfortunately breaks up during extraction, and it is not certain whether all the lens matter has been washed out. This, however, is better treated by retrobulbar injections and will be discussed under the next heading. Lastly, where the unwise use of topical steroids has led to the development of a chronic corneal ulcer, repository therapy can work wonders.

\section{Where the Lesion is Inaccessible to Topical Therapy}

The causes of posterior uveitis cannot be separated from those of anterior uveitis; thus we have either a non-granulomatous, a granulomatous, or a mixed posterior uveitis. It is harder to unravel the relative importance of the various aetiological factors in posterior than in anterior uveitis, and in the majority of cases the diagnosis is presumptive, not probable, as in anterior uveitis; in addition, the incidence of uveitis limited to the posterior segment is less, so that fewer cases were available in this study. Of those treated, only two responded well, but the result was so dramatic in the first case as to warrant continued use in all cases. This was a young man in his late twenties who had for some months been investigated for a cloudy vitreous. The condition was, and has remained, unilateral. The investigations were all negative with the exception of one of the skin tests for diagnosis of allergy. There was a marked cutaneous reaction to "dry rot", and as he was a farm worker who lived in a tied cottage in a rural area this was a credible finding. However, there was no proof that de-sensitization with the prepared vaccine healed the ocular lesion, although the condition slowly settled. Suddenly he developed an acute Jensen's juxta-papillary choroiditis in the same eye, which no form of therapy, including topical steroids, appeared to affect, the vitreous remaining filled with cells. At this point one retrobulbar injection of Depo-Medrone was given and the vitreous cleared overnight; simultaneously, the spread of choroiditis ceased. Two weeks later there was a relapse; a second retrobulbar injection was at once administered with the same immediate success. Since then the eye has been quiet, the vitreous clear of all inflammatory cells, and the area of choroid affected has not increased in size, having scarred over.

The second case treated successfully was one of those mentioned at the close of the previous section, namely a lens-induced (or phakotoxic) uveitis which almost reached the stature of an endophthalmitis phako-anaphylactica. The seriousness of this condition is indicated by the fact that de Veer (1940) reported two cases where extracapsular extraction, in which lens material had been left behind in the eye, antedated sympathetic ophthalmia in the other. It is not necessary to review the mysterious aetiology of sympathetic ophthalmia here. If it is related to the development of uveal pigment hypersensitivity then, rightly speaking, the treatment of this lesion (discussed on p. 302, Section 1) should be listed in the next section which deals with hypersensitivity reactions. Although we cannot yet be dogmatic about the aetiology of sympathetic ophthalmia, nevertheless it is clearly of vital importance to control quickly both phakotoxic uveitis and endophthalmitis phako-anaphylactica, which are 
deep-seated lesions untouched by topical therapy. In one such case where two retrobulbar injections of Depo-Medrone were given at a fortnightly interval, a violent phakotoxic uveitis caused by residual lens matter was brought under control. If there is any doubt as to whether or not lens matter has been left behind, one would appear to be justified in practising retrobulbar corticotherapy before the patient leaves the operating table. In this connexion it should be mentioned that the important clinical feature clinching the diagnosis of phakotoxic uveitis is the development of mutton-fat deposits over the back of the cornea; this indicates clearly that one is dealing with more than surgical trauma. As far as the aetiology of lens-induced uveitis is concerned, this too might be better discussed in Section 3 (below) because not only does the lesion resemble that which follows the injection of toxins into an eye, but it also resembles that which results from the contact of hypersensitive tissue with small quantities of specific antigen. Woods (1961) believes it is more likely to be the former, which is why it has been discussed in this section.

As far as retinal lesions are concerned, a limited success was achieved in a young woman with a cytoid body arteritis in systemic lupus erythematosus. The evidence admittedly is slight, based on the finding that the cytoid bodies became smaller in size and number after the injection, and this might very well have happened anyway without any treatment whatsoever. However, there is an important principle involved here, because this lesion can lead to serious defects of vision and because, as mentioned earlier, there is cumulative evidence that prolonged systemic treatment with steroids (the treatment favoured in systemic lupus erythematosus) can produce posterior subcapsular cataracts. Hughes (1964) has pointed out the possibility that the prolonged topical administration of steroid drops might also produce cataracts.

The safer potential of the more intensive short-term therapy afforded by the repository technique is well illustrated in the case just described and its wide application underlined.

Some cases of optic neuritis were treated with retrobulbar injections of DepoMedrone in this study, but only one out of four patients appeared to benefit. This was a young man who developed a unilateral optic neuritis two weeks after a decayed tooth had been extracted. Clearly, the evidence is slight, but mention is made of it here because it is a useful and simple procedure when dealing with an early optic neuritis or papillitis, the nature of which frequently takes so long to discover.

Inflammatory lesions of the lids and lacrimal passages do not as a rule require steroid therapy, but in chronic dacryocystitis, injections around or into the lacrimal sac have proved helpful; in one particularly troublesome mucocele, Depo-Medrone was placed within the sac twice at a three-week interval, and the effect was to reduce the amount of mucus on compression to a minimum. It would appear to be a useful procedure in such cases, and I now use it as a routine practice before surgery in all cases of infected sacs.

\section{To Inhibit Hypersensitivity Reactions}

It has been noted in Section 2 that sympathetic ophthalmia and lens-induced uveitis may be hypersensitivity reactions, and it has been shown that retrobulbar injections of Depo-Medrone can control these conditions. Unfortunately, their 
nature is still a matter for debate. The same may be said of the auto-immune diseases, of which three varieties were treated in this study-disseminated lupus erythematosus, pemphigus, and Stevens-Johnson disease. Nobody denies the efficacy of steroids such as Medrone and Depo-Medrone in inflammatory diseases, but the role, if any, that they play in controlling hypersensitivity reactions is on a less certain footing in ophthalmic practice, largely because of problems of classification, as in the rare conditions mentioned above. The results in the present study do little to clarify our theories concerning these unusual diseases. There is one condition, however, in which this form of therapy probably does cast some light.

An ocular lesion which is of supreme interest at the moment is meta- or postherpetiform kerato-uveitis. Is the keratitis or uveitis a hypersensitivity reaction or is it due to the presence of the virus?

In the present study, eight cases, five with stromal keratitis and three with uveitis associated with stromal keratitis, all with a known history of dendritic ulcer, were treated by repository corticotherapy. Two of the five stromal keratitis cases had a chronic open ulcer and it was discovered that they had been given topical steroids elsewhere early on; the other three keratitides were classic cases of stromal (disciform) keratitis. The five patients with stromal keratitis, even the two with open ulcers, who were given subconjunctival Depo-Medrone, all did well, but the three with uveitis did badly; in fact, two out of the three eyes were lost. It would be interesting to try Depo-Medrone injections by the retrobulbar route in the latter type of case, where the steroid is further removed from the focus of inflammation.

The effects of cortisone on the mesenchymal tissue of the cornea are well known. There is a decrease in hyperaemia, less exudation, and a diminished cellular migration and infiltration at the site of the ulcer; on the adverse side, there is retardation of the early phase of repair of corneal tissue and, after an initial liberation of excess antibody, a subsequent fall because of the suppression of antibody formation. This occurs because the fixed and mobile defence cells which move in and which are concerned with antibody formation are lysed and made ineffective. In the case of the acute dendritic ulcer, it is recognized that the effects are serious because subsequent protection of the ocular tissues depends upon the development of the antibodies; moreover, if the processes of repair are delayed the herpes virus is better able to penetrate more deeply into the stroma. With topical steroids, a high local titre produces these very effects; in this way a post-herpetiform stromal reaction is more likely to occur later on if and when more antigen reaches the antibodies deeply-placed in the stroma. The fact that Depo-Medrone benefits stromal keratitis supports the view that this condition is not due to the presence of multiplying virus in the corneal stroma but is a hypersensitivity reaction. On the opposite side of the coin, the disastrous effects of using steroids in a post-herpetiform uveitis supports the view held by some that the uveitis depends upon the actual presence of the herpes virus in the iris, and is not a hypersensitivity reaction, at least not in every case.

This whole subject of post- or meta-herpetic keratitis and uveitis is still far from clear, and for fuller information one should read Cavara and Bietti (1952), Thygeson, Hogan, and Kimura (1957), Hewson (1957), Postic and Jelesic (1958), Woods (1961), Kimura (1962), Rodger (1963), and Hughes (1964). 


\section{Summary}

(1) The various factors to be considered when placing a long-acting steroid in ocular tissues are discussed.

(2) For anterior segmental lesions, a subconjunctival injection of $0 \cdot 15 \mathrm{ml}$. of DepoMedrone directly into the lower fornix is preferred. This will act for three weeks.

(3) For posterior segmental lesions, after a retrobulbar injection of $0.50 \mathrm{ml}$. of 1-2 per cent. Lignocaine, $1.0 \mathrm{ml}$. Depo-Medrone (believed to last from two to four weeks) is injected down the same needle.

(4) A subconjunctival deposit of Depo-Medrone recovered after 21 days from a human eye was unequivocally identified as active unchanged methylprednisolone acetate.

(5) An injection of Depo-Medrone caused no appreciable harm to pieces of extrinsic ocular muscle and Tenon's capsule, the tissues being recovered fourteen days after the injections.

(6) Apart from the many practical advantages of repository corticotherapy, this method of treatment was found to be more effective than topical therapy, and safer and cheaper than systemic, in several types of lesion, especially in anterior nongranulomatous or mixed uveitis, or certain auto-immune diseases, and chronic ulcers.

(7) Where the lesion is inaccessible to topical therapy, as in posterior uveitis, or inflammatory conditions of the lacrimal passages, the repository technique is admirably suited.

(8) The role of steroids in controlling hypersensitivity reactions is applied to the theories surrounding the aetiology of sympathetic ophthalmia, phakotoxic uveitis, some of the auto-immune diseases, and post-herpetiform keratitis and uveitis. In the case of the latter, the author concludes that post-herpetiform keratitis is a hypersensitivity reaction, but the uveitis is not, because the keratitis alone responds favourably to Depo-Medrone:

Messrs. Upjohn Ltd. are sincerely thanked for supplying the Depo-Medrone used in this study, and also for carrying out the biochemical assays and preparation of histological specimens recorded on p. 300 .

\section{REFERENCES}

BONNET, M. (1962). J. Méd. Lyon, 43, 1937.

BOYD, B. F. (1960). Highlts Ophthal. (U.S. edition), 3, 278.

Cavara, V., and BietTI, G. B. (1952). "Le manifestazioni oculari delle malattie da virus e da rickettsie". Cappelli, Bologna.

Coles, R. S., Krohn, D. L., Breslin, H., and Braunstein, R. (1962). Amer. J. Ophthal., 54, 407.

Crews. S. J., (1963). Brit. med. J., 1, 1644.

DE VeER, J. A. (1940). Arch. Ophthal. (Chicago), 23, 237.

Flandre, O., Damon, M., Weisbuch, J. J., Peillex, F., François, P., and Ferran, J. L. (1963). Thérapie, $18,1237$.

GeBERTT, S. (1961). Lancet, 2, 344.

Hewson, G. E. (1957). Irish J. med. Sci., 6th ser., no. 380, p. 372.

HUGHES, W. F. (1964). "Year Book of Ophthalmology 1963-4," pp. 131, 223. Year Book Publishers, Chicago.

KimuRA, S. J. (1962). Trans. Amer. ophthal. Soc., 60, 440.

Klodell, C. B., and Bloomberg, L. (1964). Eye, Ear, Nose Thr. Monthly, 43, 51.

Postic, S., and Jelesic, Z. (1958). Bull. Soc. franç. ophtal., 71, 202.

RODGER, F. C. (1963). Trans. ophthal. Soc. U.K., 83, 637.

Thygeson, P., Hogan, M. J., and Kimura, S. J. (1957). Trans. Amer. Ophthal. Soc., 55, 333.

Woods, A. C. (1961). "Endogenous Inflammations of the Uveal Tract", p. 211. Williams and Wilkins, Baltimore. 